\title{
ISO AND LABORATORY ASTROPHYSICS
}

\author{
L.J. ALLAMANDOLA \\ NASA-Ames Research Center \\ Mountain View, CA 94035 \\ USA
}

ISO, the Infrared Space Observatory is capable of measuring spectra across most of the mid-infrared, the region from 4000 to $500 \mathrm{~cm}^{-1}(2.5-20 \mu \mathrm{m})$. Of these $3500 \mathrm{~cm}^{-1}$, about 670 have been inaccessible to astronomers due to strong atmospheric absorptions and about $750 \mathrm{~cm}^{-1}$ require an airborne platform, making access limited. ISO will provide high quality spectra in the $19 \%$ of the celestial mid-infrared which has never been studied and dramatically increase the number of spectra in the $21 \%$ with limited access. Thus ISO has the potential to nearly double our knowledge of the mid-IR spectroscopic properties of the cosmos. This knowledge, in turn, will revolutionize our understanding of its chemical make-up because of the mid-infrared's powerful compositional diagnostic capabilities.

In order to fully exploit this potential requires that laboratory data are available with which one can analyze the spectra ISO measures. To maximize the scientific return from this limited lifetime mission it is particularly important to have as much data available as possible before launch in order to plan an intelligent follow-up program before the cryogen runs out. This dictates that laboratory studies focus on the spectral properties of astrophysically relevant materials with important bands in the new regions and regions of limited access.

As I am most familiar with the IR absorption spectra of dense and diffuse clouds and with IR emission spectra associated with aromatics, I will summarize the work needed in order to be prepared for the discoveries in these areas. The table summarizes what potentially will be probed. As with all pioneering spectroscopic studies unexpected features will be found. These can be recognized only if archives of the appropriate laboratory data are available.

To interpret the absorption spectra infrared spectroscopic studies should be carried out on realistic astrophysical ice analogs containing the species and subgroups listed in the third column of the table. For the emission bands, studies are needed on isolated, neutral and ionized polycyclic aromatic hydrocarbons which are hydrogenated (PAHs) and deuterated (PADs).

With the appropriate laboratory data, the absorption spectra ISO obtains will shed light on $\mathrm{D} / \mathrm{H}$ ratios, composition, abundances, and chemical processing of ices and dust in dense molecular clouds, the diffuse interstellar medium and in cold disks. In many cases gas phase column densities of the same molecules can be measured along the same line of sight. Emission spectra will shed light on $\mathrm{PAH} \mathrm{D/H}$ ratios and compositional variations as a function of object type along the H-R diagram and the relationship of PAHs (if any) to the IR cirrus. 
TABLE OF WAVELENGTH REGIONS AND TARGET BANDS RELATED TO INTERSTELLAR DUST WHICH WILL BE PIONEERED BY ISO.

\begin{tabular}{|c|c|c|c|}
\hline \multicolumn{2}{|c|}{ OPAQUE REGION } & \multicolumn{2}{|c|}{ TARGET BAND CARRIERS } \\
\hline Wavelength $(\mu \mathrm{m})$ & Obscurer & Absorption & Emission \\
\hline $2.5-2.75$ & {$\left[\mathrm{H}_{2} \mathrm{O}\right]$} & Free $\mathrm{H}_{2} \mathrm{O}$ & $?$ \\
\hline $4.1-4.4$ & {$\left[\mathrm{CO}_{2}\right]$} & $\begin{array}{l}\mathrm{CD}, \mathrm{OD}, \mathrm{CO}_{2}, \\
\mathrm{C} \equiv \text { Nstretches }\end{array}$ & PAD:CD stretch \\
\hline$* 5.1-9$ & $\begin{array}{c}\mathrm{H}_{2} \mathrm{O}, \mathrm{CH}_{4} \\
\mathrm{~N}_{\mathrm{x}} \mathrm{O}_{\mathrm{y}}, \ldots\end{array}$ & $\begin{array}{l}\text { Organics } \\
>\mathrm{C}=\mathrm{O}\end{array}$ & $\begin{array}{c}\text { PAH:C-Cstretch } \\
\text { (most intense) }\end{array}$ \\
\hline $9.5-10$ & {$\left[\mathrm{O}_{3}\right]$} & $\mathrm{O}_{3}$ & $?$ \\
\hline $14-16$ & {$\left[\mathrm{H}_{2} \mathrm{O}, \mathrm{CO}_{2}\right]$} & $\begin{array}{c}\mathrm{CD}, \mathrm{OD}, \mathrm{O}_{3}, \mathrm{CO}_{2} \\
=\mathrm{C}-\mathrm{H} \text { bends }\end{array}$ & PAD:CD bend \\
\hline$>50$ & {$\left[\mathrm{H}_{2} \mathrm{O}\right]$} & Ice phonons & PAH plane bends \\
\hline
\end{tabular}

* Limited access - requires airborne platform. 